\title{
THE PRESENT STATUS OF THE DECAYING NEUTRINO THEORY
}

\author{
D.W. SCIAMA \\ International School for Advanced Studies (SISSA), Trieste, \\ International Centre for Theoretical Physics, Trieste, Italy \\ Department of Physics, Oxford, UK
}

A discussion is given of recent observations relevant to the decaying neutrino theory. The HST spectrum of the halo star HD 93521 obtained and analysed by Spitzer and Fitzpatrick supports our predictions that warm opaque clouds in the Galaxy should possess substantial partial ionisation, and that the electron density in such clouds near the sun should be constant.

A recent stringent upper limit on the intergalactic ionising flux at $z \sim 0$ obtained by Vogel et al. requires us to reduce the energy of decay photons to less than $13.8 \mathrm{eV}$. The decay flux at $z \geq 2$ could still be mainly responsible for the ionisation of Lyman $\alpha$ clouds and of the intergalactic medium at such red shifts. A further consequence of the Vogel et al. result is that decay photons can no longer ionise nitrogen.

Recent estimates of the isotropic cosmic background at $1500 \AA$ by Henry and Murthy and by Witt and Petersohn agree well with the predictions of the decaying neutrino theory.

A satellite experiment will be carried out in 1995 to search for the predicted decay line from neutrinos within a parsec of the sun. The detectors are being built by Bowyer and his colleagues at the Center for EUV Astrophysics in Berkeley and will be launched in a Spanish minisatellite (principal investigator C. Morales).

\section{Introduction}

I am very grateful to the organisers of this Symposium for the opportunity to bring my decaying neutrino theory up to date. This theory was originally proposed to explain the widespread ionisation of hydrogen in the Milky Way, which at the time was puzzling astronomers (Sciama 1990, 1993a). I suggested that if most of the dark matter in our Galaxy consists 
of neutrinos with non-zero rest mass, then decay photons from these neutrinos might be the main ionising source for the interstellar medium. This hypothesis is relevant to our present Symposium because it turns out that the decay lifetime required could make the cosmological distribution of neutrinos the main ionising source for the pregalactic medium, the intergalactic medium and Lyman $\alpha$ clouds (Sciama 1993a). Thus the hypothesis, while speculative, is strongly unifying. In addition it makes a number of rather precise predictions, and is therefore very vulnerable to observational disproof. Nevertheless it remains a viable theory at the present time.

In this talk I shall describe the impact on the theory of a number of recent or still unpublished observations. They are the following:

a) The HST spectrum of the halo star HD 93521 (Spitzer and Fitzpatrick 1993).

b) $\mathrm{H}_{\alpha}$ observations of the intergalactic HI cloud $1225+01$ (Vogel et al. 1994).

c) A probable Gunn-Peterson absorption trough in HeII (Jakobsen et al. 1994).

d) The isotropic extragalactic background at $1500 \AA$ (Henry and Murthy 1993, Witt and Petersohn 1994).

e) A future satellite experiment to search for the predicted decay line from neutrinos within $\sim 1$ parsec of the sun.

\section{The basic idea}

Particle physicists tell us that if neutrinos have a non-zero rest-mass, then a neutrino of type 1 ( $\operatorname{say} \tau$ ) would be expected to decay into a photon and a neutrino of type 2 ( say $\mu$ ) of lower mass. The decay lifetime cannot yet be predicted, since it depends strongly on unknown details of particle physics models. We would thus have the decay

$$
\nu_{1} \rightarrow \gamma+\nu_{2} \text {. }
$$

Conservation of energy and momentum tell us that the energy $E_{\gamma}$ of the decay photon in the rest frame of the parent neutrino is given by

$$
E_{\gamma}=\frac{1}{2} m_{1}\left(1-\frac{m_{2}^{2}}{m_{1}^{2}}\right) .
$$

Hence the process produces a line, a fact of great importance for the subsequent analysis. It is likely that $m_{1} \gg m_{2}$, in which case

$$
E_{\gamma} \approx \frac{1}{2} m_{1},
$$


which we shall assume from now on. In order to ionise hydrogen we need

$$
E_{\gamma} \geq 13.6 \mathrm{eV}
$$

Hence

$$
m_{1} \geq 27.2 \mathrm{eV} .
$$

We may compare this condition with the Tremaine-Gunn phase space constraint for neutrinos of type 1 to dominate the mass of the Galaxy. One obtains (Sciama 1993a)

$$
m_{1} \geq 27.6 \mathrm{eV},
$$

which is essentially the same condition. One also notices that one is immediately in the cosmologically interesting range for $m_{1}$.

We now consider the required value of the decay lifetime $\tau$. This was evaluated in Sciama (1990) from the condition that the decay photons should be the main source of the interstellar density of free electrons. One obtains

$$
\tau \approx 2-3 \times 10^{23} \mathrm{secs},
$$

which is about a million times longer than the age of the universe.

This estimate for $\tau$ would conflict with the analysis by Davidsen at al. (1991) of their HUT observations of the rich cluster of galaxies A665, which led to $\tau>2 \times 10^{24}$ secs. However a re-analysis by Melott et al, (1994) using an n-body simulation of cluster formation in the presence of neutrinos, modified this constraint to $\tau>2 \times 10^{23}$ secs, which is just compatible with our required value.

\section{The HST observations of HD 93521}

In order to prepare for these observations we first consider two basic predictions of the decaying neutrino theory. These predictions apply to an interstellar cloud which is opaque to photons with energy $E_{\gamma}$. Essentially every decay photon produced in the cloud ionises an $\mathrm{H}$ atom in the cloud which then recombines. We thus have in a steady state

$$
\frac{n_{\nu}}{\tau}=\alpha n_{e}^{2},
$$

where $n_{\nu}$ is the neutrino density in the cloud, $n_{e}$ is the electron density and $\alpha$ is the recombination coefficient. For clouds within 1 or $2 \mathrm{kpc}$ of the sun $n_{\nu}$ would be nearly constant (halo population). For warm clouds with $T \sim 10^{4} \mathrm{~K}, \alpha$ would be nearly constant. Hence $n_{e}$ is nearly constant, that 
is, is independent of the total gas density in the cloud. This is our first prediction.

The second prediction follows immediately from the fact that we have a strong source of ionisation uniformly distributed throughout an opaque cloud. We thus expect to find substantial partial ionisation within such an opaque cloud. This would not be expected in the prevailing theory (Miller and Cox 1993, Domgorgen and Mathis 1994, Dove and Shull 1994) in which UV photons from distant $\mathrm{O}$ stars are regarded as the main source of ionisation. In this case only the outer skins of opaque clouds could be ionised (apart from a small contribution to the inner ionisation from carbon atoms and from cosmic rays). In addition the electron density would then be governed by the total density of the skins, which need not be nearly constant.

We now consider the observations of Spitzer and Fitzpatrick (1993). Their HST spectrum of HD 93521 revealed four opaque slowly moving clouds which are unlikely to be disturbed by shock waves. They derived the electron density $n_{e}$ in each cloud from the observed column density of CII ions in the upper excited fine-structure level of the ground state.

They found that

a) $n_{e}$ is the same in each cloud (with only a $10 \%$ scatter about the mean)

b) the ionised gas in each cloud is mixed up with the opaque neutral gas, rather than being confined to its surface. Thus the clouds seem to be partially ionised in their interiors. The agreement of these results with the predictions of the neutrino decay theory was pointed out and analysed by Sciama (1993b).

\section{The intergalactic ionising flux}

A new more stringent upper limit on the intergalactic ionising flux $F$ at $z \sim 0$ can be derived from recent $\mathrm{H}_{\alpha}$ observations of the intergalactic HI cloud $1225+01$ (Vogel et al. 1994). This limit is

$$
F_{o b s} \leq 1.6 \times 10^{5} \text { photons } \mathrm{cm}^{-2} \mathrm{sec}^{-1} .
$$

This result imposes a new stringent upper limit on the energy $E_{\gamma}$ of a decay photon. To see this we compute the contribution $F_{\nu}$ to $F$ from the cosmological distribution of decaying neutrinos. To allow for the effect of the red shift, which limits the volume of integration, we put

$$
E_{\gamma}=13.6+\epsilon \mathrm{eV} .
$$

Then

$$
F=\frac{n_{\nu}}{\tau} \frac{c}{H_{0}} \frac{\epsilon}{13.6}
$$


where $n_{\nu}$ is here the cosmological neutrino density at $z \sim 0$ and $c / H_{0}$ is the radius of the universe. With $n_{\nu} \approx 100 \mathrm{~cm}^{-3}, \tau \approx 2 \times 10^{23} \mathrm{sec}$ and $c / H_{0} \approx 2 \times 10^{28} \mathrm{~cm}$ (see later) we deduce that

$$
\epsilon<0.2 \mathrm{eV} .
$$

Hence

$$
E_{\gamma}<13.8 \mathrm{eV},
$$

and

$$
m_{1}<27.6 \mathrm{eV} \text {. }
$$

Accordingly

$$
m_{1}=27.4 \pm 0.2 \mathrm{eV},
$$

which constrains $m_{1}$ to better than 1 percent.

If we allow for the contribution to the density of the universe from baryons, according to the standard model of big bang nucleosynthesis, we find that

$$
\Omega h^{2}=0.28 \pm 0.003
$$

where $H_{0}=100 \quad h \quad \mathrm{~km} \mathrm{sec} \mathrm{spc}^{-1}$. If the cosmological constant $\lambda$ is zero, then arguments concerning the age of the universe (Sciama 1993a) show that

$$
\Omega \approx 1
$$

and

$$
H_{0} \approx 54 \mathrm{~km} \mathrm{sec}^{-1} \mathrm{Mpc}^{-1} .
$$

This value for $H_{0}$ is close to that advocated by Tammann at this Symposium. If $\Omega=1$ exactly, then our uncertainty in $H_{0}$ is less than

$$
\pm \frac{1}{2} \mathrm{~km} \mathrm{sec}^{-1} \mathrm{Mpc}^{-1} \text {. }
$$

We now consider the implications of this theory for the ionising flux at large $z$. With $\Omega \sim 1$ we are close to the Einstein-de Sitter model in which

$$
F(z)=(1+z)^{3 / 2} F(0) .
$$

Hence, for example,

$$
F_{\nu}(2)<8 \times 10^{5} \mathrm{~cm}^{-2} \mathrm{sec}^{-1} .
$$


According to the proximity effect for Lyman $\alpha$ clouds, as discussed at this Symposium by Bechtold

$$
F_{\nu}(2) \sim 5 \times 10^{5} \mathrm{~cm}^{-2} \mathrm{sec}^{-1} .
$$

The contribution to $F(2)$ from quasars is still uncertain, but some estimates lead to a value a few times less than this. It is thus still possible that decay photons make the main contribution to $F(2)$, so long as $F_{\nu}$ is close to its present upper limit.

The absence of a Gunn-Peterson absorption trough in HI could also be mainly due to $F_{\nu}$, but this depends on the presently uncertain density of the intergalactic medium. This question has recently been illuminated by the important probable discovery of a Gunn-Peterson absorption trough in HeII (Jakobsen et al. 1994) which was reported on at this Symposium by Jakobsen. The implied lower limit on the ratio $J_{H I} / J_{H e I I}$ of the effective intergalactic ionising fluxes at the HI and HeII edges can be converted to a lower limit on the ratio $\Phi_{H I} / \Phi_{H e I I}$ of the emissivity of the sources by allowing for absorption in the intergalactic medium and in Lyman $\alpha$ clouds and Lyman limit systems (Madau and Meiksin 1994). If this ratio is found to be too steep to be due to quasars it would indicate that a source which preferentially ionises $\mathrm{HI}$ is making a major contribution to $J_{\mathrm{HI}}$. This would fit in nicely with the decaying neutrino theory, but more observational work is needed before this possibility can be assessed.

\section{The ionisation of nitrogen}

One important consequence of our new upper limit on $E_{\gamma}$ is that decay photons can no longer ionise nitrogen (whose threshold is at $14.5 \mathrm{eV}$ ). We must therefore rediscuss the ionisation of nitrogen observed in the local interstellar medium, throughout the Galaxy, and in NGC 891, which we previously attributed to decay photons (Sciama 1993a). Since this Symposium is devoted to cosmology, we do not discuss this question here in detail, and will return to it elsewhere. We simply remark that the large column density of NII towards $\beta \mathrm{CMa}$ implied by the observations of Gry et al. (1985) can be attributed to radiation from $\beta \mathrm{C} M \mathrm{Ma}$ itself. The reason for this is that the column density of HI towards $\beta \mathrm{CMa}$ is $2 \times 10^{18} \mathrm{~cm}^{-2}$ (Drew 1994) whereas that towards the nearby star $\beta \mathrm{CMa}$ is only $9 \times 10^{17} \mathrm{~cm}^{-2}$ (Cassinelli et al. 1994 , Vallerga and Welsh 1994). This disparity indicates that an additional cloud is present along the line of sight towards $\beta \mathrm{CMa}$, and since the ionising flux in this direction is about ten times greater than in neighbouring directions, it is natural to attribute this extra flux to the additional cloud lying suitably close to $\beta \mathrm{CMa}$. This extra flux would then be responsible for most of the ionisation of nitrogen in this direction. 
With these few remarks we leave the subject of the ionisation of nitrogen and return to it elsewhere.

\section{The cosmic background at $1500 \AA$}

The earliest lower limits on the lifetime $\tau$ of a decaying neutrino were based on observational estimates of the cosmic background in the far ultra-violet (Stecker 1980, Kimble, Bowyer and Jakobsen 1981). Today the value of the observed cosmic background is somewhat controversial (Jakobsen, this Symposium). The most recent estimates for an isotropic background at $1500 \AA$ (Henry and Murthy 1993, Witt and Petersohn 1994) are

$$
300 \pm 80 \text { photons } \mathrm{cm}^{-2} \mathrm{sec}^{-1} \text { ster }^{-1} \AA^{-1} \text { (C.U.) }
$$

The most recent value (Armand et al. 1994) for the contribution due to galaxies is

$$
40-120 \text { C.U. }
$$

The red shifted contribution from decay photons for $\tau \sim 2 \times 10^{23}$ secs is

$$
200 \text { C.U. }
$$

Thus the decaying neutrino theory is close to being tested by these observations.

\section{A future satellite experiment}

It is planned to search for the predicted decay line from neutrinos near the sun using detectors on board a satellite. The sun is known to be immersed in a cloud with $n_{\mathrm{HI}} \sim 0.1 \mathrm{~cm}^{-3}$, so that unit optical depth for decay photons would occur at a distance $\sim 0.5 \mathrm{pc}$. The resulting decay flux at the Earth would then be about 600 photons $\mathrm{cm}^{-2} \mathrm{sec}$. Bowyer and his colleagues at the Center for EUV Astrophysics in Berkeley are building detectors with sufficient sensitivity and energy resolution to observe such a flux. The equipment will be flown on board a Spanish minisatellite (Principal Investigator Carmen Morales) and launch is planned for the end of 1995. The observation period will be about a year, and we hope to obtain a result in time to report it at the next General Assembly of the IAU.

\section{References}

Armand, C., Milliard, B., and Deharveng, J.M. (1994), $A$ \& $A, \mathbf{2 8 4}, 12$. Cassinelli, J.P. et al. (1994), to be published.

Davidsen, A.F. et al. (1991), Nature 351, 128. 
Domgorgen, H. and Mathis, J.S. (1994), Ap. J. 428, 647.

Dove, J.B. and Shull, J.M. (1994), Ap. J. 430, 222.

Drew, J.E. (1994), private communication.

Gry, C., York, D.G. and Vidal-Majdar, A. (1985), Ap. J. 296, 593.

Henry, R.C. and Murthy, J. (1993), Ap. J. 418, L17.

Jakobsen, P. et al. (1994), Nature 370, 35.

Kimble, R., Bowyer, S. and Jakobsen, P. (1981), Phys. Rev. Lett. 46, 80.

Madau, P. and Meiksin, A. (1994), to be published.

Melott, A.L., Splinter, R.F., Persic, M. and Salucci, P. (1994), Ap. J. 416, 12.

Miller, W.W. and Cox D.P. (1993), Ap. J. 417, 579.

Sciama, D.W. (1990), Ap. J. 364, 54.

Sciama, D.W. (1992), Int. Journ. of Mod. Phys. D. 1, 161.

Sciama, D.W. (1993a), Modern Cosmology and the Dark Matter Problem, Cambridge University Press.

Sciama, D.W. (1993b), Ap. J., 409, L25.

Stecker, F.W. (1980), Phys. Rev. Lett., 45, 1460.

Spitzer, L. and Fitzpatrick, E.L. (1993), Ap. J., 409, 299.

Vallerga, J.V. and Welsh, B.Y. (1994), to be published.

Vogel, S.N., Weymann, R., Rauch, M. and Hamilton, T. (1994), to be published.

Witt, A.N. and Petersohn, J.K. (1994), in The First Symposium on the Infrared Cirrus and Diffuse Interstellar Clouds, ASP Conference Series Vol. 58, ed. R.M. Cutri and W.B. Latter. 